\title{
Extracellular Matrix and Biomineralization
}

\author{
J.L. Arias, and M.S. Fernández
}

Faculty of Veterinary and Animal Sciences and Center for Advanced Interdisciplinary Research in Materials (CIMAT), Universidad de Chile, Santiago, Chile.(jarias@uchile.cl)

Modern technologies require innovative approaches for controlled fabrication of crystalline materials with complex forms and novel properties [1-4]. Biomineralization is a widespread phenomenon among living systems (e.g., egg and mollusk shells, crustacean carapaces, echinoderm exoskeleton and spines, sponge spicules, pearls, corals, bones, and teeth) [5-7], which leads to the formation of precisely controlled inorganic-organic composites, where the minute extracellular matrix component exerts substantial control on the mineralization process, resulting in the formation of particles of uniform size, novel crystal morphology, specific crystallographic orientation, and interesting properties [5-11]. Understanding the mechanisms that regulate the fabrication of such highly ordered biocomposite ceramics may provide procedures for the synthesis of novel high-performance composite materials. Therefore, biomimetic design for the production of advanced composites has been explored [12-16]

Biomineralization have been extensively studied in carbonate-based structures. More than 20 different proteins have been described to be involved in the control of biomineralization of egg- and seashells, crustacean carapaces, echinoderm spines and spicules, pearls, etc [17]. However, no enough similarities among their aminoacid sequences have been found when such proteins were analysed. If there is no enough sequence homology between these proteins to propose a simplified mechanism of biomineralization control, what other features have the different models in common? By studying egg- and seashells, barnacles, corals and echinoderm structures we have found the persistant occurrence of specific sulfated macromolecules referred to as proteoglycans. These macromolecules have a particular calcium affinity and behave as gels. The distribution of these proteoglycans together with their function on in vitro mineralization assays, showed that they are involved not only in the nucleation but also in the growth of the calcium carbonate crystalline phase [18-21]. Such proteoglycans (probably together with the particular proteins already described), conform a gel compartment were mineralization takes place. However this compartment is limited by other extracellular matrix macromolecules (such as type X collagen or chitin) which supports and structures the gel but do not participate directly in the mineralization process.

A commonly used strategy in shell biomineralization is the elaboration of a well organized extracellular organic matrix, which regulates where, when, and in what form mineralization will occur [1]. From the comparative studies of the structure and formation of carbonate-based structures, it is possible to propose a four-step mechanism of biomineralization consisting of a precise spatio-temporal arrangement of sequentially deposited macromolecules [22]. The first step is the fabrication of an inert laminar substrate or framework which compartmentalizes a microenvironment where mineralization will take place. This scaffolding consists of a non-mineralized, well-ordered hydrophobic organic material and usually is composed of $\beta$-chitin, type X collagen, or other not well characterized biopolymers. The second step is the fabrication of particular polyanionic macromolecules, which are deposited on the previously formed inert scaffolding, and where nucleation of the calcium crystals takes place. These macromolecules are aspartate- or 
glutamate-rich proteins or keratan sulfate-rich proteoglycans. The third step is the fabrication of a gel structure consisting of silk fibroin-like proteins or proteoglycans and containing acidic proteins or dermatan sulfate. This gel not only controls polymorphism, but also the diffusion-controlled growth, face-growing rates and habit of the crystal formed. The fourth step is the arrest of crystal formation and is related to the fabrication of new inert scaffolding or the deposition of particular hydrophobic inhibitory proteins (e.g., the eggshell cuticle). These four steps open a new field for designing synthetic processes to build new bio-inspired composites with desirable properties [23].

\section{References}

[1] A.H. Heuer et al., Science 255 (1992) 1098

[2] J.D. Hartgerink et al., Science 294 (2001) 1684

[3] B.C. Bunker et al., Science 264 (1994) 48

[4] S. Mann, Angew. Chem. Int. Ed. 39(2000) 3392

[5] H.A. Lowenstam and S. Weiner, On Biomineralization, Oxford University Press, U.K., 1989

[6] S. Mann. Biomineralization, Oxford University Press, U.K., 2001

[7] K. Simkiss, and K.M. Wilbur, Biomineralization: Cell Biology and Mineral Deposition, Academic Press, San Diego, CA, 1989

[8] Y. Nys et al., Poultry \& Avian Biol.Rev. 10 (1999) 142

[9] C. A. Orme et al., Nature 411 (2001) 775

[10] B.L. Smith et al., Nature 399 (1999) 761

[11] R.Z. Wang et al., J. Mater. Res. 16 (2001) 2485

[12] J. Aizenberg et al., Nature 398 (1999) 495

[13] G. Xu et al., J. Am. Chem. Soc. 123 (2001) 2196

[14] N. Almqvist et al., Mater. Sci. Engin. C 7 (1999) 37

[15] B.L. Zhou, Mater. Sci. Engin. C 11 (2000) 13

[16] M. Sarikaya, PNAS 96 (1999) 14183

[17] H. Nagasawa, Thalassas (2003)

[18] J.L. Arias et al., Inter. Rev. Cytol. 145 (1993) 217

[19] M.S. Fernande et al.,. Matrix Biol. 19( 2001) 793

[20] J.L. Arias et al., In Biomineralization, formation, diversity, evolution and application, $\mathrm{H}$. Kozawa and I. Kobayashi, eds., Niigata Univ. Press (2003).

[21] M.S. Fernandez et al., MRS Symp. Proc. 724 (2002)

[22] J.L. Arias and M.S. Fernandez, Mat. Charact. (2003)

[23] This research was supported by CONICYT under grant FONDAP 11980002. 